\title{
DISCOVERY OF A REFLECTION DUST ENVELOPE AROUND IRC+10216
}

\author{
M. Tamura ${ }^{1}$ \\ T. Hasegawa ${ }^{2}, \mathrm{~N}$. Ukita ${ }^{2}$ \\ I. Gatley ${ }^{3}$, I. S. McLean ${ }^{3}$, M. G. Burton ${ }^{3}$ \\ J. T. Rayner ${ }^{4}$, and M. J. McCaughrean ${ }^{4}$ \\ ${ }^{2}$ Nobeyama Radio Observatory, Tokyo Astronomical Observatory, \\ ${ }^{4}$ Department of Astronomy, University of Edinburgh, U.K..
}

${ }^{1}$ Department of Physics, Kyoto University, Japan. University of Tokyo, Japan.

${ }^{3}$ United Kingdom Infrared Telescope Unit, Hawaii.

Infared polarimetric and photometric mapping observations at $\mathrm{K}(2.2 \mu \mathrm{m})$ and $\mathrm{H}(1.65 \mu \mathrm{m})$ have revealed an extended dust envelope around the late-type star IRC +10216 . The observations were made on the 3.8-m United Kingdom Infrared Telescope on Mauna Kea, Hawai, in 1985 December and 1987 January and February. The polarization observations were made by emplying the Kyoto polarimeter (Sato et al. 1987). Great care was taken to check the contamination by stray light in the telescope and instruments as the source on peak was extremely bright $(\mathrm{K} \sim 0 \mathrm{mag})$. From the observations of normal stars, we found that the polarized intensity (degree of polarization times the intensity) was a good measure of the envelope, free from contamination by stray light, although the intensity and the degree of polarization suffered from the contamination separately.

Figure 1 shows the polarization vectors at $K$ superposed on the surface brightness map and Figure 2 shows the radial distribution of the polarized intensity. The infrared envelope is roughly circular symmetric with radial extent greater than 1 arcmin $(\sim 0.09 \mathrm{pc})$, comparable to the size of the molecular gas 
envelope (Bieging, Chapman, and Welch 1984, wilson, Schwartz, and Epstein 1973, wannier et al. 1979, wootten et al. 1982). The polarization vectors show a clear centro-symmetric pattern, indicating that the infrared nebulosity is due to scattering by dust grains in the envelope. The radial distribution of dust grains derived from Figure 2 is most likely to obey an inversesquare law, suggesting a steady mass loss between $r \sim 15^{\prime \prime}$ and $60^{\prime \prime}$.

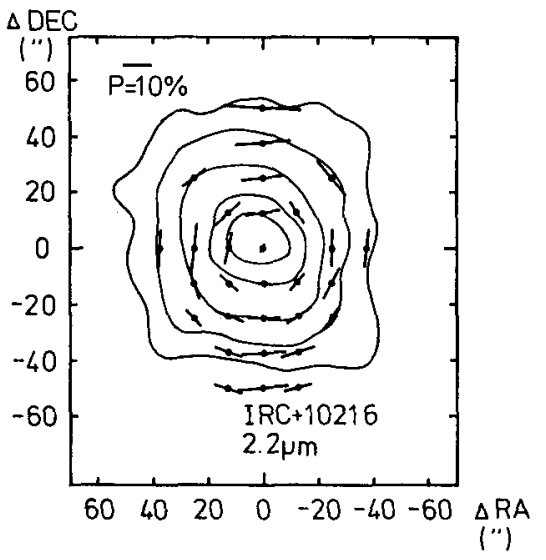

Figure 1: Surface brightness and polarization map of IRC +10216 in the $\mathrm{K}$ band, with the beam size of $20^{\prime \prime}$ and $12^{\prime \prime}$, respectively. Filled and open circles in the middle of the polarization vectors indicate positions of polarimetry made in 1987 January and February, respectively.

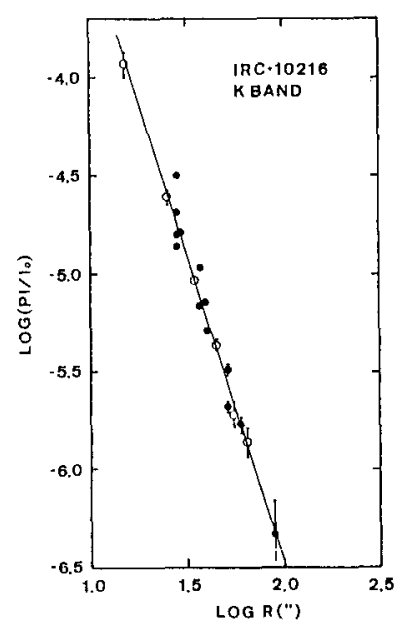

Figure 2: Logarithmic radial distribution of the polarized intensity of the envelope of IRC +10216 in the $K$ band.

Open and closed circles are data taken with different photometers on different days.

A least-square-fitted line is shown by a thin line.

\section{REFERENCES}

Bieging, J.H., Chapman, B., and welch, W.J. 1984 , Ap. J., 285, 656 .

Sato, S. et al. 1987, M.N.R.A.S., in press.

Wannier, P.G., Leighton, R.B., Knapp, G.R., Redman, R.O., Phillips, T.G., and Huggins, P.J. 1979, Ap. J., 230, 149.

Wilson, w.J., Schwartz, P.R., and Epstein, E.E. 1973 , Ap. J., 183, 871.

Wootten, A., Lichten, S.M., Sahai, R., and Wannier, P.G. 1982, Ap. J., 257, 151 . 